\title{
Dynamics of stochastic hybrid Gilpin-Ayala system with impulsive perturbations
}

\author{
Ruihua Wu \\ College of Science, China University of Petroleum (East China), Qingdao 266555, P. R. China.
}

Communicated by S. H. Wu

\begin{abstract}
This paper is mainly concerned with the dynamics of the stochastic Gilpin-Ayala model under regime switching with impulsive perturbations. The goal is to analyze the effects of Markov chain and impulse on the dynamics. Some asymptotic properties are considered and sufficient criteria for stochastic permanence, extinction, non-persistence in the mean and weak persistence are obtained. The critical value among the extinction, non-persistence in the mean and weak persistence is explored. Our results demonstrate that the dynamics of the model have close relations with the impulse and the stationary distribution of the Markov chain. (C)2017 All rights reserved.
\end{abstract}

Keywords: Gilpin-Ayala model, Markov chain, impulsive perturbations, stochastic permanence, extinction. 2010 MSC: 34F05, 34D05.

\section{Introduction and preliminaries}

The Gilpin-Ayala model, is denoted by the following ordinary differential equation

$$
\dot{x}(t)=x(t)\left[b-a x^{\lambda}(t)\right], \quad \lambda>0,
$$

and $\lambda$ is the parameter to modify the classical logistic model. In the model, $x(t)$ expresses the population size at time $t, b$ denotes the intrinsic growth rate and $b / a$ is the carrying capacity. Many good results have been reported on model $(1.1)$, see $[4,6]$ and the references cited therein.

If the environmental noise, which is important in ecosystem (see e.g. Gard [7, 8]), is taken into account, we get the corresponding Itô equation

$$
d x(t)=x(t)\left[b-a x^{\lambda}(t)\right] d t+\sigma_{1} x(t) d B_{1}(t)+\sigma_{2} x^{1+\lambda}(t) d B_{2}(t) .
$$

In the model, $\sigma_{i}^{2}(i=1,2)$ denotes the intensity of the noise, the pair $B(t)=\left(B_{1}(t), B_{2}(t)\right)^{\top}$ is a twodimensional Brownian motion defined on a complete probability space $\left(\Omega, \mathcal{F},\left\{\mathcal{F}_{t}\right\}_{t} \geqslant 0, \mathbb{P}\right)$ with a filtration

Email address: wu_ruihua@hotmail.com (Ruihua $\mathrm{Wu}$ )

doi:10.22436/jnsa.010.02.10 
$\left\{\mathcal{F}_{t}\right\}_{t \geqslant 0}$ satisfying the usual conditions. The stochastic Gilpin-Ayala models have been studied a lot, the readers can refer to $[12,13,19,22,23]$ and the references therein.

Further, increasing attention has been devoted to hybrid systems, in which continuous dynamics are intertwined with discrete events. One of the distinct features of such systems is that the underlying dynamics are subject to changes with respect to certain configurations. It is recognized that using a continuous-time Markov chain is suitable to depict such kind of systems where they differ by factors such as rain falls or nutrition $[5,33]$.

Let $r(t), t \geqslant 0$, be a right-continuous Markov chain taking values in a finite state space $S=\{1,2, \cdots, N\}$ with generator $Q=\left(q_{i j}\right)_{N \times N}$ given by

$$
\mathbb{P}=\{r(t+\triangle t)=j \mid r(t)=i\}=\left\{\begin{array}{cl}
q_{i j} \Delta t+o(\triangle t), & \text { if } j \neq i \\
1+q_{i i} \Delta t+o(\Delta t), & \text { if } j=i
\end{array}\right.
$$

where $\triangle t>0, q_{i j} \geqslant 0$ is the transition rate from $i$ to $j$ if $i \neq j$ while $\sum_{j=1}^{N} q_{i j}=0$. Throughout the paper, we assume that $B(t)$ is independent of $r(t)$. Further assume that the Markov chain $r(t)$ is irreducible which means that the system can switch from any regime to any other regime. And the irreducibility implies that the Markov chain has a unique stationary (probability) distribution $\pi=\left(\pi_{1}, \pi_{2}, \cdots, \pi_{N}\right) \in \mathbb{R}^{1 \times N}$ (see [2]) satisfying

$$
\pi \mathrm{Q}=0,
$$

subject to $\sum_{i=1}^{N} \pi_{i}=1$ and $\pi_{i}>0$, for all $i \in S$.

Introducing the Markovian switching into model (1.2), we get the general stochastic hybrid system, or system under regime switching:

$$
d x(t)=x(t)\left[b(r(t))-a(r(t)) x^{\lambda}(t)\right] d t+\sigma_{1}(r(t)) x(t) d B_{1}(t)+\sigma_{2}(r(t)) x^{1+\theta}(t) d B_{2}(t), \quad \theta>0 .
$$

In its operation, the hybrid system will switch from one mode to another according to the law of the Markov chain. If the initial state $r(0)=i \in S$, then (1.4) obeys

$$
d x(t)=x(t)\left[b(i)-a(i) x^{\lambda}(t)\right] d t+\sigma_{1}(i) x(t) d B_{1}(t)+\sigma_{2}(i) x^{1+\theta}(t) d B_{2}(t),
$$

till time $\tau_{1}$ when the Markov chain switches to $r\left(\tau_{1}\right)=j \in S$ from $r(0)$, then the system obeys

$$
d x(t)=x(t)\left[b(j)-a(j) x^{\lambda}(t)\right] d t+\sigma_{1}(j) x(t) d B_{1}(t)+\sigma_{2}(j) x^{1+\theta}(t) d B_{2}(t),
$$

until the next switching. The system will continue to switch as long as the Markov chain switches. The Markov chain has important impacts on the population dynamics. Takeuchi et al. [34] revealed the significant effect of Markovian switching on the population system: both its subsystems develop periodically but switching between them makes them become neither permanent nor dissipative.

Let us take a more further step, in reality, due to some natural or man-made factors, the growth of species often undergoes some discrete changes of relatively short time interval at some fixed times, such as drought, flooding, hunting, planting, etc. These phenomena cannot be considered continually, so in this case, system (1.4) fails to explain these phenomena. Introducing the impulsive effects into the model may be more suitable for such phenomena, see $[3,14]$.

Lots of deterministic population dynamical systems with impulsive effects have been proposed and studied. Many results on dynamical behavior for such systems have been published, see e.g. [1, 9, 11, 36] and the references therein. Recently, authors of [16-18,32] considered the stability of stochastic differential equation (SDE) with impulsive effects. However, so far as we know, there are few papers published which study the impulsive stochastic population model, see [24, 25, 35]. By now, there are no result related to the general stochastic Gilpin-Ayala system under regime switching with impulsive effects. 
Inspired by the above discussions, in this paper, we study the general stochastic hybrid system with impulsive effects, or system under regime switching:

$$
\left\{\begin{aligned}
d x(t)= & x(t)\left[b(r(t))-a(r(t)) x^{\lambda}(t)\right] d t+\sigma_{1}(r(t)) x(t) d B_{1}(t) \\
& +\sigma_{2}(r(t)) x^{1+\theta}(t) d B_{2}(t), \quad t \neq t_{k}, \quad k \in \mathbb{N} \\
x\left(t_{k}^{+}\right)- & x\left(t_{k}\right)=b_{k} x\left(t_{k}\right), \quad k \in \mathbb{N},
\end{aligned}\right.
$$

where $\lambda>0, \theta>0$.

It is worth pointing out that many classical and important stochastic models are the special cases of (1.5). If $\lambda=1, \sigma_{2}(r(t)) \equiv 0$, system (1.5) is the model (2) for nonautonomous case in [26]. If $\lambda=\theta=1$, system (1.5) becomes the model (5) in [24]. This indicates that our model is more general and meaningful.

As we know that, the extinction and stochastic permanence are two important and interesting topics in the biomathematics, and the threshold value of persistence and extinction is meaningful in practice. So in this paper, we focus on exploring the effects of the Markovian switching and impulse on the extinction and persistence of system (1.5). The rest of the paper is arranged as follows. In Section 2 we propose sufficient conditions for the existence of the global positive solutions. We give the critical value between persistence and extinction in Section 3. In Section 4, we establish sufficient criteria for stochastic permanence by using the theory of M-matrices. The numerical simulations are illustrated to confirm our results in Section 5. We conclude our paper with further remarks in Section 6.

\section{Global positive solutions}

Throughout this paper, we assume $\min _{k \in S} a(k)>0$. For a matrix or vector $G, G \gg 0$ means all elements of $G$ are positive, and the symbol $Z^{N \times N}$ denotes $Z^{N \times N}:=\left\{A=\left(a_{i j}\right)_{N \times N}: a_{i j} \leqslant 0, i \neq j\right\}$. Take $\prod_{i=1}^{n} y_{i}(t)=y_{1}(t) \cdots y_{n}(t)$ and assume that a product equals unity if the number of factors is zero.

In the following, for convenience and simplicity, we use the following notations:

$$
\hat{f}=\min _{k \in S} f(k), \quad \check{f}=\max _{k \in S} f(k), \quad f^{*}=\limsup _{t \rightarrow+\infty} f(t), \quad f_{*}=\liminf _{t \rightarrow+\infty} f(t), \overline{f(t)}=t^{-1} \int_{0}^{t} f(s) d s .
$$

In view of the biological significance of model (1.5), only positive solutions are meaningful. Before we analyze the properties of the solutions, first we should ensure the existence of positive solutions. The following theorem illustrates this point.

Theorem 2.1. For any initial value $\mathrm{r}(0) \in \mathrm{S}$ and $\mathrm{x}(0)>0$, (1.5) admits a unique global positive solution $\mathrm{x}(\mathrm{t})$ on $t \geqslant 0$ a.s. (almost surely).

Proof. Consider the following Itô equation without impulse:

$$
\begin{aligned}
d y(t)= & y(t)\left[b(r)-a(r) \prod_{0<t_{k}<t}\left(1+b_{k}\right)^{\lambda} y^{\lambda}(t)\right] d t+\sigma_{1}(r) y(t) d B_{1}(t) \\
& +\sigma_{2}(r) \prod_{0<t_{k}<t}\left(1+b_{k}\right)^{\theta} y^{1+\theta}(t) d B_{2}(t)
\end{aligned}
$$

with initial value $y(0)=x(0)$. Here for simplicity, we drop $t$ from $b(r(t))$ and $a(r(t))$ etc. According to the result of [22, Theorem 1], (2.1) has a unique global positive solution $y(t)$ on $t \geqslant 0$. Let $x(t)=$ $\prod_{0<t_{k}<t}\left(1+b_{k}\right) y(t)$, then we claim that $x(t)$ is the solution of (1.5) with initial data $x(0)$. We easily see that $x(t)$ is continuous on $\left(0, t_{1}\right)$ and each interval $\left(t_{k}, t_{k+1}\right) \subset[0, \infty), k \in \mathbb{N}$. And for $t \neq t_{k}$

$$
\mathrm{d} x(\mathrm{t})=\mathrm{d}\left[\prod_{0<\mathrm{t}_{\mathrm{k}}<\mathrm{t}}\left(1+\mathrm{b}_{\mathrm{k}}\right) \mathrm{y}(\mathrm{t})\right]=\prod_{0<\mathrm{t}_{\mathrm{k}}<\mathrm{t}}\left(1+\mathrm{b}_{\mathrm{k}}\right) \mathrm{d} \mathrm{y}(\mathrm{t})
$$




$$
\begin{aligned}
= & \prod_{0<t_{k}<t}\left(1+b_{k}\right) y(t)\left[b(r)-a(r) \prod_{0<t_{k}<t}\left(1+b_{k}\right)^{\lambda} y^{\lambda}(t)\right] d t \\
& +\sigma_{1}(r) \prod_{0<t_{k}<t}\left(1+b_{k}\right) y(t) d B_{1}(t)+\sigma_{2}(r) \prod_{0<t_{k}<t}\left(1+b_{k}\right)^{1+\theta} y^{1+\theta}(t) d B_{2}(t) \\
= & x(t)\left[b(r)-a(r) x^{\lambda}(t)\right] d t+\sigma_{1}(r) x(t) d B_{1}(t)+\sigma_{2}(r) x^{1+\theta}(t) d_{2}(t) .
\end{aligned}
$$

Further, for every $t_{k} \in \mathbb{R}_{+}, k \in \mathbb{N}$,

$$
\begin{aligned}
x\left(t_{k}^{+}\right) & =\lim _{t \rightarrow t_{k}^{+}} x(t)=\lim _{t \rightarrow t_{k}^{+}} \prod_{0<t_{j}<t}\left(1+b_{j}\right) y(t)=\prod_{0<t_{j} \leqslant t_{k}}\left(1+b_{j}\right) y\left(t_{k}^{+}\right) \\
& =\left(1+b_{k}\right) \prod_{0<t_{j}<t_{k}}\left(1+b_{j}\right) y\left(t_{k}\right)=\left(1+b_{k}\right) x\left(t_{k}\right),
\end{aligned}
$$

and

$$
x\left(t_{k}^{-}\right)=\lim _{t \rightarrow t_{k}^{-}} x(t)=\lim _{t \rightarrow t_{k}^{-}} \prod_{0<t_{j}<t}\left(1+b_{j}\right) y(t)=\prod_{0<t_{j}<t_{k}}\left(1+b_{j}\right) y\left(t_{k}^{-}\right)=\prod_{0<t_{j}<t_{k}}\left(1+b_{j}\right) y\left(t_{k}\right)=x\left(t_{k}\right) .
$$

This completes the proof.

Now, we conclude that system (1.5) admits a unique global positive solution. However, from the biological point of view, the non-explosion property and positivity in a population dynamical system are often not good enough. Further, in the next we will investigate asymptotic properties of the solutions.

\section{Critical value between extinction and persistence}

In the sequel, we will consider the long time behaviors of the positive solutions which are important in practice, because they can predict the future properties of the solutions. First we give several concepts, then we will attempt to illustrate sufficient conditions for them.

Definition 3.1 ([22]). Let $x(t)$ be the solution of (1.5),

(a) if $\lim _{t \rightarrow+\infty} x(t)=0$ a.s., then species $x(t)$ is said to be extinct;

(b) if $\lim _{t \rightarrow+\infty} \overline{\chi^{\lambda}(t)}=\lim _{t \rightarrow+\infty} t^{-1} \int_{0}^{t} x^{\lambda}(s) d s=0$ a.s., then species $x(t)$ is said to be non-persistent in the mean;

(c) if $x^{*}=\limsup _{t \rightarrow+\infty}(t)>0$ a.s., then species $x(t)$ is said to be weakly persistent.

Definition 3.2 ([13]). The solution $x(t)$ of $(1.5)$ is said to be stochastically permanent, if for any $\varepsilon \in(0,1)$, there is a pair of positive constants $\mathrm{H}_{1}=\mathrm{H}_{1}(\varepsilon)$ and $\mathrm{H}_{2}=\mathrm{H}_{2}(\varepsilon)$ such that

$$
\liminf _{t \rightarrow+\infty} \mathbb{P}\left[x(t) \leqslant H_{1}\right] \geqslant 1-\varepsilon \text {, and } \liminf _{t \rightarrow+\infty} \mathbb{P}\left[x(t) \geqslant H_{2}\right] \geqslant 1-\varepsilon,
$$

where $x(t)$ is an arbitrary solution of the equation with initial value $x(0)>0, r(0) \in S$.

From the definitions we can see that extinction implies non-persistence in the mean, and the stochastic permanence is the strongest property, we will consider them one by one. First, we give an asymptotic pathwise estimation.

Theorem 3.3. For any initial value $x(0)>0$ and $r(0) \in S$, the solution $x(t)$ of (1.5) obeys

$$
\limsup _{t \rightarrow \infty} \frac{\ln x(t)}{t} \leqslant \bar{h}:=\limsup _{t \rightarrow \infty} \frac{\sum_{0<t_{k}<t} \ln \left(1+b_{k}\right)}{t}+\sum_{i=1}^{N} \pi_{i} h(i) \quad \text { a.s.. }
$$

Particularly, if $\overline{\mathrm{h}}<0$, then species $x(\mathrm{t})$ will go to extinction, where $\mathrm{h}(\mathfrak{i})=\mathrm{b}(\mathfrak{i})-0.5 \sigma_{1}^{2}(\mathfrak{i})$. 
Proof. Applying generalized Itô formula to (2.1) leads to

$$
\begin{aligned}
\mathrm{d} \ln y(t)= & {\left[\mathrm{h}(\mathrm{r})-\mathrm{a}(\mathrm{r}) \prod_{0<\mathrm{t}_{\mathrm{k}}<\mathrm{t}}\left(1+\mathrm{b}_{\mathrm{k}}\right)^{\lambda} \mathrm{y}^{\lambda}(\mathrm{t})-0.5 \sigma_{2}^{2}(r) \prod_{0<\mathrm{t}_{k}<\mathrm{t}}\left(1+\mathrm{b}_{\mathrm{k}}\right)^{2 \theta} \mathrm{y}^{2 \theta}(\mathrm{t})\right] \mathrm{dt} } \\
& +\sigma_{1}(\mathrm{r}) \mathrm{dB}_{1}(\mathrm{t})+\sigma_{2}(\mathrm{r}) \prod_{0<\mathrm{t}_{\mathrm{k}}<\mathrm{t}}\left(1+\mathrm{b}_{\mathrm{k}}\right)^{\theta} \mathrm{y}^{\theta}(\mathrm{t}) \mathrm{dB}_{2}(\mathrm{t}),
\end{aligned}
$$

which is equivalent to

$$
\begin{aligned}
\ln y(t)= & \ln y(0) \\
& +\int_{0}^{t}\left[h(r(s))-a(r(s)) \prod_{0<t_{k}<s}\left(1+b_{k}\right)^{\lambda} y^{\lambda}(s)-0.5 \sigma_{2}^{2}(r(s)) \prod_{0<t_{k}<s}\left(1+b_{k}\right)^{2 \theta} y^{2 \theta}(s)\right] d s \\
& +M_{1}(t)+M_{2}(t)
\end{aligned}
$$

where $M_{1}(t)=\int_{0}^{t} \sigma_{1}(r(s)) d B_{1}(s), M_{2}(t)=\int_{0}^{t} \sigma_{2}(r(s)) \prod_{0<t_{k}<s}\left(1+b_{k}\right)^{\theta} y^{\theta}(s) d B_{2}(s)$. The quadratic variation of $M_{1}(t)$ is

$$
\left\langle M_{1}, M_{1}\right\rangle(t)=\int_{0}^{t} \sigma_{1}^{2}(r(s)) d s \leqslant \check{\sigma}_{1}^{2} t .
$$

Using the strong law of large numbers for martingales results in

$$
\lim _{t \rightarrow \infty} \frac{M_{1}(t)}{t}=0 \text { a.s.. }
$$

The quadratic variation of $M_{2}(t)$ is $\left\langle M_{2}, M_{2}\right\rangle(t)=\int_{0}^{t} \sigma_{2}^{2}(r(s)) \prod_{0<t_{k}<s}\left(1+b_{k}\right)^{2 \theta} y^{2 \theta}(s) d s$. By exponential martingale inequality,

$$
\mathbb{P}\left(\sup _{0 \leqslant \mathrm{t} \leqslant n}\left[M_{2}(t)-\frac{1}{2}\left\langle M_{2}, M_{2}\right\rangle(t)\right]>2 \ln n\right) \leqslant \frac{1}{n^{2}} .
$$

By applying Borel-Cantelli lemma we see that for almost all $\omega \in \Omega$, there is a random integer $n_{0}=n_{0}(\omega)$ such that if $n \geqslant n_{0}$,

$$
M_{2}(t) \leqslant 2 \ln n+\frac{1}{2} \int_{0}^{t} \sigma_{2}^{2}(r(s)) \prod_{0<t_{k}<s}\left(1+b_{k}\right)^{2 \theta} y^{2 \theta}(s) d s
$$

holds for all $0 \leqslant t \leqslant n, n \geqslant n_{0}$. Using above inequality to (3.1) leads to

$$
\ln y(t) \leqslant \ln y(0)+\int_{0}^{t}\left[h(r(s))-a(r(s)) \prod_{0<t_{k}<s}\left(1+b_{k}\right)^{\lambda} y^{\lambda}(s)\right] d s+2 \ln n+M_{1}(t),
$$

for all $0 \leqslant t \leqslant n, n \geqslant n_{0}$. Recalling that $x(t)=\prod_{0<t_{k}<t}\left(1+b_{k}\right) y(t)$, we obtain

$$
\ln x(t) \leqslant \ln x(0)+\sum_{0<t_{k}<t} \ln \left(1+b_{k}\right)+\int_{0}^{t} h(r(s)) d s-\int_{0}^{t} a(r(s)) x^{\lambda}(s) d s+2 \ln n+M_{1}(t) .
$$

Then for $n-1 \leqslant t \leqslant n, n \geqslant n_{0}$, we derive

$$
\frac{\ln x(t)}{t} \leqslant \frac{\ln x(0)}{t}+\frac{1}{t} \sum_{0<t_{k}<t} \ln \left(1+b_{k}\right)+\frac{1}{t} \int_{0}^{t} h(r(s)) d s+\frac{2 \ln n}{n-1}+\frac{M_{1}(t)}{t} .
$$

By (3.2) and the ergodic property of the Markov chain, we conclude our desired assertion. This completes the proof. 
Remark 3.4. It is evident that $x(t) \equiv 0$ is the trivial solution of (1.5). By Theorem 3.3 we conclude that if $\bar{h}=\limsup _{t \rightarrow \infty} t^{-1} \sum_{0<t_{k}<t} \ln \left(1+b_{k}\right)+\sum_{i=1}^{N} \pi_{i} h(i)<0$, the trivial solution of system (1.5) is almost surely exponentially stable, it also demonstrates that the species will go extinct exponentially fast.

Remark 3.5. If $\limsup _{\mathrm{t} \rightarrow \infty} \mathrm{t}^{-1} \sum_{0<\mathrm{t}_{\mathrm{k}}<\mathrm{t}} \ln \left(1+\mathrm{b}_{\mathrm{k}}\right)=0$, then our result is consistent with [22, Theorem 2]. It reveals that the small impulse has no nature impact on the species, but from the result we can see that the negative impulse can contribute on the extinction of the population and the positive impulse can resist the extinction of species, this also coincides with the reality.

Theorem 3.6. If $\bar{h} \geqslant 0$, then $x(t)$ satisfies

$$
\limsup _{t \rightarrow \infty} \frac{1}{t} \int_{0}^{t} x^{\lambda}(s) \mathrm{d} s \leqslant \bar{h} / \hat{a} \text { a.s.. }
$$

Particularly, if $\overline{\mathrm{h}}=0$, then species $x(\mathrm{t})$ modeled by (1.5) is non-persistent in the mean.

Proof. Note that for for all $\varepsilon>0$, there exists a constant $\mathrm{T}>0$ such that

$$
\frac{\ln x(0)}{\mathrm{t}} \leqslant \frac{\varepsilon}{4}, \frac{1}{\mathrm{t}}\left[\sum_{0<\mathrm{t}_{\mathrm{k}}<\mathrm{t}} \ln \left(1+\mathrm{b}_{\mathrm{k}}\right)+\int_{0}^{\mathrm{t}} \mathrm{h}(\mathrm{r}(\mathrm{s}))\right] \mathrm{d} s \leqslant \overline{\mathrm{h}}+\frac{\varepsilon}{4}, \frac{2 \ln \mathrm{n}}{\mathrm{t}} \leqslant \frac{\varepsilon}{4}, \frac{\mathrm{M}_{1}(\mathrm{t})}{\mathrm{t}} \leqslant \frac{\varepsilon}{4},
$$

for $0<T<n-1 \leqslant t \leqslant n, n \geqslant n_{0}$. By above inequalities, (3.3) becomes into

$$
\ln x(t) \leqslant(\varepsilon+\bar{h}) t-\hat{a} \int_{0}^{t} x^{\lambda}(s) d s .
$$

By [27, Lemma 4], we have $\limsup _{t \rightarrow \infty} t^{-1} \int_{0}^{t} x^{\lambda}(s) d s \leqslant(\varepsilon+\bar{h}) / \hat{a}$ a.s.. By the arbitrariness of $\varepsilon$, we get our required assertion. This completes the proof.

Theorem 3.7. If $\overline{\mathrm{h}}>0$, then species modeled by system (1.5) will be weakly persistent.

Proof. To get the weak persistence, we need to show $x^{*}>0$ a.s. If this assertion is not true, then $\mathbb{P}(E)>0$, where $E=\left\{\limsup _{t \rightarrow+\infty} x(t)=0\right\}$. By (3.1), we see that

$$
\begin{aligned}
\frac{\ln x(t)}{t}= & \frac{\ln x(0)}{t}+\frac{1}{t}\left[\sum_{0<t_{k}<t} \ln \left(1+b_{k}\right)+\int_{0}^{t} h(r(s)) d s\right]-\frac{1}{t} \int_{0}^{t} a(r(s)) x^{\lambda}(s) d s \\
& -\frac{1}{2 t} \int_{0}^{t} \sigma_{2}^{2}(r(s)) x^{2 \theta}(s) d s+\frac{M_{1}(t)}{t}+\frac{M_{2}(t)}{t} .
\end{aligned}
$$

Note that for all $\omega \in E, \lim _{t \rightarrow+\infty} x(t, \omega)=0$. Therefore,

$$
\limsup _{t \rightarrow \infty} \frac{\ln x(t, \omega)}{t} \leqslant 0, \quad \lim _{t \rightarrow \infty} \frac{1}{t} \int_{0}^{t} a(r(s)) x^{\lambda}(s) d s=0, \lim _{t \rightarrow \infty} \frac{1}{t} \int_{0}^{t} \sigma_{2}^{2}(r(s)) x^{2 \theta}(s) d s=0, \quad \lim _{t \rightarrow+\infty} \frac{M_{2}(t)}{t}=0 .
$$

Substituting above inequalities into (3.4) and using (3.2) we can get a contradiction

$$
0 \geqslant \limsup _{t \rightarrow \infty} t^{-1} \ln x(t, \omega)=\bar{h}>0 .
$$

This completes the proof.

Remark 3.8. Theorems 3.3, 3.6, and 3.7 have a clear and interesting biological interpretation. It is not difficult to see that the extinction and persistence of species $x(t)$ modeled by (1.5) depend only on the value of $\bar{h}$, which also implies that the long time behaviors of system (1.5) have close relations with the impulse and stationary distribution of the Markovian switching. 
Remark 3.9. Let us consider the effects of white noise on the species. By the expression $h(i)=b(i)-$ $0.5 \sigma_{1}^{2}(i)$, we can see that the white noise $\sigma_{1}(r(t))$ imposed on the intrinsic growth rate $b(r(t))$ contributes to the extinction of the species and has negative effect on the survival of the population. The white noise $\sigma_{2}(r(t))$ imposed on the intraspecific competition coefficient has no impact on the extinction and persistence of the species, which coincides with the special case when $\lambda=\theta=1$ (see [24]).

Remark 3.10. Let us look at the effects of impulse on the extinction and persistence of species. If $b_{k}$ is big enough such that $\limsup _{t \rightarrow \infty} t^{-1} \sum_{0<t_{k}<t} \ln \left(1+b_{k}\right)>\sum_{i=1}^{N} \pi_{i} h(i)$, that is $\bar{h}>0$, then species will be weakly persistent which means that the positive impulse is always advantageous for the existence of the species, e.g. planting. Further, we can see that the positive impulse can resist the impact of the white noise which accords with the reality and will not happen in the model without impulse.

Remark 3.11. Let us consider some subsystem

$$
\left\{\begin{array}{l}
d x(t)=x(t)\left[b(i)-a(i) x^{\lambda}(t)\right] d t+\sigma_{1}(i) x(t) d B_{1}(t)+\sigma_{2}(i) x^{1+\theta}(t) d B_{2}(t), \quad t \neq t_{k}, k \in \mathbb{N}, \\
x\left(t_{k}^{+}\right)-x\left(t_{k}\right)=b_{k} x\left(t_{k}\right), \quad k \in \mathbb{N},
\end{array}\right.
$$

for the state $i$. Similarly, we can prove that if $\bar{h}(i):=\limsup _{t \rightarrow \infty} t^{-1} \sum_{0<t_{k}<t} \ln \left(1+b_{k}\right)+h(i)<0$, then species $x(t)$ of (3.5) will go extinct, if $\bar{h}(i)=0$, then species $x(t)$ of (3.5) will be non-persistent in the mean, if $\bar{h}(i)>0$, then species $x(t)$ of (3.5) will be weakly persistent, see [28].

Remark 3.12. Let us turn to see the impacts of the Markovian switching on the system. Here we take an example for negative impulse. If $h(i)<0$, for every $i \in S$, then every subsystem (3.5) is extinct. From Theorem 3.3 we can see that, in this case, as a result of Markovian switching, the overall behavior of (1.5) also remains extinct. However, Theorems 3.3, 3.6, and 3.7 indicate an interesting result: if some individual subsystems are extinctive, the corresponding $h(i)>0$ or $h(i)<0$, some are persistent, the corresponding $h(i)$ must be positive, due to the Markovian switching, the value $\bar{h}$ may be less than zero or large than zero, that is to say, the overall system may be extinct or persistent.

\section{Stochastic permanence}

Stochastic permanence is a more important asymptotic behavior, it implies that the population will survive forever, so it is interesting and valuable in biomathematics. In the following, we propose the conditions under which the species is stochastically permanent. First we talk about the moment property.

Assumption 4.1. There exist two positive constants $m$ and $M$ such that $m \leqslant \prod_{0<t_{k}<t}\left(1+b_{k}\right) \leqslant M$ for all $t>0$.

Lemma 4.2. Under Assumption 4.1, for any $\mathrm{p} \in(0,1)$, there exists a constant $\mathrm{K}$ such that the solution of (1.5) has the property

$$
\limsup _{t \rightarrow+\infty} \mathbb{E}\left[x^{p}(t)\right] \leqslant K .
$$

Proof. The proof follows Luo and Mao [29]. For any $p \in(0,1)$, define $V(y)=y^{p}$. For any $y(0)<k$, define a stopping time

$$
\sigma_{k}=\inf \{t \geqslant 0, y(t)>k\} .
$$

Then $\sigma_{k} \uparrow \infty$ a.s. as $k \rightarrow \infty$. Applying generalized Itô's formula reaches that

$$
\begin{aligned}
\mathrm{d}\left[e^{t} V(y)\right]= & e^{t} y^{p}\left\{1+p\left[b(r)-a(r) \prod_{0<t_{k}<t}\left(1+b_{k}\right)^{\lambda} y^{\lambda}+0.5(p-1) \sigma_{1}^{2}(r)\right.\right. \\
& \left.\left.+0.5(p-1) \sigma_{2}^{2}(r) \prod_{0<t_{k}<t}\left(1+b_{k}\right)^{2 \theta} y^{2 \theta}\right]\right\} d t \\
& +p e^{t} y^{p}\left[\sigma_{1}(r) d_{1}(t)+\sigma_{2}(r) \prod_{0<t_{k}<t}\left(1+b_{k}\right)^{\theta} y^{\theta} d B_{2}(t)\right]
\end{aligned}
$$




$$
\begin{aligned}
& \leqslant e^{t} y^{p}\left[1+p \check{b}-p a m^{\lambda} y^{\lambda}+0.5 p(p-1) \hat{\sigma}_{2}^{2} m^{2 \theta} y^{2 \theta}\right] d t \\
& +p e^{t} y^{p}\left[\sigma_{1}(r) d B_{1}(t)+\sigma_{2}(r) \prod_{0<t_{k}<t}\left(1+b_{k}\right)^{\theta} y^{\theta} d B_{2}(t)\right] \\
& \leqslant e^{t} K_{1}+p e^{t} y^{p}\left[\sigma_{1}(r) d_{1}(t)+\sigma_{2}(r) \prod_{0<t_{k}<t}\left(1+b_{k}\right)^{\theta} y^{\theta} d_{B}(t)\right],
\end{aligned}
$$

where $K_{1}$ is a positive constant. Integrating above inequality and then taking expectations for both sides, one can arrive at

$$
\mathbb{E}\left[e^{t \wedge \sigma_{k}} V\left(y\left(t \wedge \sigma_{k}\right)\right)\right] \leqslant y^{p}(0)+\mathbb{E} \int_{0}^{t \wedge \sigma_{k}} e^{s} K_{1} d s
$$

Letting $k \rightarrow \infty$ leads to $\mathbb{E}\left[e^{t} y^{p}(t)\right] \leqslant y^{p}(0)+K_{1} e^{t}$. Further, we have $\limsup _{t \rightarrow+\infty} \mathbb{E}\left[y^{p}(t)\right] \leqslant K_{1}$. By now, we can conclude that $\limsup _{t \rightarrow+\infty} \mathbb{E}\left[x^{p}(t)\right]=\limsup _{t \rightarrow+\infty} \mathbb{E}\left[\prod_{0<t_{k}<t}\left(1+b_{k}\right)^{p} y^{p}(t)\right] \leqslant M^{p} K_{1}:=K$ which is our desired assertion. This completes the proof.

In the following, we use the theory of M-matrices to get the stochastic permanence. First we give a condition for the generator $\mathrm{Q}$ of the Markov chain.

Assumption 4.3. For some $u \in S, \mathrm{q}_{i u}>0, \forall i \neq u$.

Lemma 4.4. Let Assumption 4.3 hold, if $\tilde{\mathrm{h}}=\sum_{\mathrm{k}=1}^{\mathrm{N}} \pi_{\mathrm{k}} \mathrm{h}(\mathrm{k})>0$, then there exists a constant $\alpha \in(0,1)$ such that the matrix

$$
A(\alpha):=\operatorname{diag}\left(\xi_{1}(\alpha), \xi_{2}(\alpha), \cdots, \xi_{N}(\alpha)\right)-Q,
$$

is a nonsingular M-matrix, where $\xi_{k}(\alpha)=\alpha(1+\theta) h(k)-0.5 \alpha^{2}(1+\theta)^{2} \sigma_{1}^{2}(k)$.

Proof. The proof of this lemma is similar to [23, Lemma 3], the reader can refer to it, here we omit the proof.

We are now in position to present our main result of this section. In the following, we write $y(t)$ with $y, V(y(t), r(t))$ with $V(y, k)$ and etc. for simplicity.

Theorem 4.5. Under Assumptions 4.1 and 4.3 together with $0<\theta \leqslant 1$ and $0<\lambda \leqslant 1+\theta$, if

$$
\tilde{\mathrm{h}}=\sum_{\mathrm{k}=1}^{\mathrm{N}} \pi_{\mathrm{k}} \mathrm{h}(\mathrm{k})>0,
$$

then species $x(t)$ modeled by (1.5) will be stochastically permanent.

Proof. First we prove $\liminf _{t \rightarrow+\infty} \mathbb{P}\left[x(t) \geqslant H_{2}\right] \geqslant 1-\varepsilon$. Define $V_{1}(y)=1 / y^{1+\theta}$, by Itô formula we derive that

$$
\begin{aligned}
d V_{1}(y(t))= & (1+\theta) V_{1}\left[a(r(t)) \prod_{0<t_{k}<t}\left(1+b_{k}\right)^{\lambda} y^{\lambda}-b(r(t))\right] d t+0.5(1+\theta)(2+\theta) \sigma_{1}^{2}(r(t)) V_{1} d t \\
& +0.5(1+\theta)(2+\theta) \sigma_{2}^{2}(r(t)) \prod_{0<t_{k}<t}\left(1+b_{k}\right)^{2 \theta} y^{\theta-1} d t-(1+\theta) \sigma_{1}(r(t)) V_{1} d B_{1}(t) \\
& -(1+\theta) \sigma_{2}(r(t)) \prod_{0<t_{k}<t}\left(1+b_{k}\right)^{\theta} y^{-1} d B_{2}(t) .
\end{aligned}
$$

For $\alpha$ given in Lemma 4.4, by [31, Theorem 2.10], there exists a vector $\vec{p}=\left(p_{1}, p_{2}, \cdots, p_{N}\right)^{\top} \gg 0$ such that $A(\alpha) \vec{p} \gg 0$ which is equivalent to

$$
p_{k}\left[\alpha(1+\theta)\left(b(k)-0.5 \sigma_{1}^{2}(k)\right)-0.5 \alpha^{2}(1+\theta)^{2} \sigma_{1}^{2}(k)\right]-\sum_{j=1}^{N} q_{k j} p_{j}>0,
$$

for $1 \leqslant k \leqslant N$. Define function $V_{2}: \mathbb{R}_{+} \times S \rightarrow \mathbb{R}_{+}$by

$$
V_{2}(y, k)=p_{k}\left(1+V_{1}\right)^{\alpha} \text {. }
$$


Making use of the generalized Itô formula yields that

$$
\begin{aligned}
d V_{2}(y(t), k)= & L_{2}(y, k) d t-\alpha p_{k}(1+\theta) \sigma_{1}(k)\left(1+V_{1}(t)\right)^{\alpha-1} V_{1}(t) d B_{1}(t) \\
& -\alpha p_{k}(1+\theta) \sigma_{2}(k) \prod_{0<t_{k}<t}\left(1+b_{k}\right)^{\theta}\left(1+V_{1}(t)\right)^{\alpha-1} y^{-1} d B_{2}(t)
\end{aligned}
$$

where

$$
\begin{aligned}
& \operatorname{LV}_{2}(y, k) \\
& =\alpha p_{k}\left(1+V_{1}\right)^{\alpha-2}\left\{\left(1+V_{1}\right)(1+\theta) V_{1}\left(a(k) \prod_{0<t_{k}<t}\left(1+b_{k}\right)^{\lambda} y^{\lambda}-b(k)\right)\right. \\
& +0.5(1+\theta)(2+\theta) \sigma_{1}^{2}(k)\left(1+V_{1}\right) V_{1} \\
& +0.5(1+\theta)(2+\theta) \sigma_{2}^{2}(k)\left(1+V_{1}\right) \prod_{0<t_{k}<t}\left(1+b_{k}\right)^{2 \theta} y^{\theta-1}+0.5(\alpha-1)(1+\theta)^{2} \sigma_{1}^{2}(k) V_{1}^{2} \\
& \left.+0.5(\alpha-1)(1+\theta)^{2} \sigma_{2}^{2}(k) \prod_{0<t_{k}<t}\left(1+b_{k}\right)^{2 \theta} y^{-2}\right\}+\sum_{j=1}^{N} q_{k j} p_{j}\left(1+v_{1}\right)^{\alpha} \\
& =\alpha p_{k}\left(1+V_{1}\right)^{\alpha-2}\left\{( 1 + V _ { 1 } ) \left[(1+\theta) V_{1}\left(a(k) \prod_{0<t_{k}<t}\left(1+b_{k}\right)^{\lambda} y^{\lambda}-b(k)\right)+0.5(1+\theta)(2+\theta) \sigma_{1}^{2}(k) V_{1}\right.\right. \\
& \left.+0.5(1+\theta)(2+\theta) \sigma_{2}^{2}(k) \prod_{0<t_{k}<t}\left(1+b_{k}\right)^{2 \theta} y^{\theta-1}\right]+0.5(\alpha-1)(1+\theta)^{2} \sigma_{1}^{2}(k) V_{1}^{2} \\
& \left.+0.5(\alpha-1)(1+\theta)^{2} \sigma_{2}^{2}(k) \prod_{0<t_{k}<t}\left(1+b_{k}\right)^{2 \theta} y^{-2}\right\}+\left(1+v_{1}\right)^{\alpha} \sum_{j=1}^{N} q_{k j} p_{j} \\
& =\left(1+V_{1}\right)^{\alpha-2}\left\{-\left[\alpha p_{k}(1+\theta) h(k)-0.5 \alpha^{2} p_{k}(1+\theta)^{2} \sigma_{1}^{2}(k)-\sum_{j=1}^{N} q_{k j} p_{j}\right] V_{1}^{2}\right. \\
& +\left[-\alpha p_{k}(1+\theta) b(k)+0.5 \alpha p_{k}(1+\theta)(2+\theta) \sigma_{1}^{2}(k)+2 \sum_{j=1}^{N} q_{k j} p_{j}\right] v_{1} \\
& +\sum_{j=1}^{N} q_{k j} p_{j}+\alpha p_{k}(1+\theta) a(k) \prod_{0<t_{k}<t}\left(1+b_{k}\right)^{\lambda} v_{1} y^{\lambda-1-\theta}+\alpha p_{k}(1+\theta) a(k) \prod_{0<t_{k}<t}\left(1+b_{k}\right)^{\lambda} y^{\lambda-1-\theta} \\
& +0.5 \alpha p_{k}(1+\theta)(2+\theta) \sigma_{2}^{2}(k) \prod_{0<t_{k}<t}\left(1+b_{k}\right)^{2 \theta} y^{\theta-1} \\
& +0.5 \alpha p_{k}(1+\theta)(2+\theta) \sigma_{2}^{2}(k) \prod_{0<t_{k}<t}\left(1+b_{k}\right)^{2 \theta} y^{-2} \\
& \left.+0.5 p_{k} \alpha(\alpha-1)(1+\theta)^{2} \sigma_{2}^{2}(k) \prod_{0<t_{k}<t}\left(1+b_{k}\right)^{2 \theta} y^{-2}\right\} \text {. }
\end{aligned}
$$

Now choose sufficiently small $\eta>0$ satisfying

$$
\alpha p_{k}(1+\theta)\left(b(k)-0.5 \sigma_{1}^{2}(k)\right)-0.5 \alpha^{2} p_{k}(1+\theta)^{2} \sigma_{1}^{2}(k)-\sum_{j=1}^{N} q_{k j} p_{j}-\eta p_{k}>0,
$$

for all $1 \leqslant k \leqslant N$. Set $V_{3}(y, k)=e^{\eta t} V_{2}(y, k)$. Using generalized Itô formula derives that

$$
\mathbb{E}\left[\mathrm{V}_{3}(\mathrm{y}(\mathrm{t}), \mathrm{r}(\mathrm{t}))\right]=\mathrm{V}_{2}(\mathrm{y}(0), r(0))+\mathbb{E} \int_{0}^{t} e^{\eta \mathrm{s}}\left[\operatorname{LV}_{2}(\mathrm{y}(\mathrm{s}), \mathrm{r}(\mathrm{s}))+\eta \mathrm{V}_{2}(\mathrm{y}(\mathrm{s}), \mathrm{r}(\mathrm{s}))\right] \mathrm{ds},
$$


where

$$
\begin{aligned}
& L V_{2}(y, k)+\eta V_{2}(y, k)=\left(1+V_{1}\right)^{\alpha-2}\left\{-\left[\alpha p_{k}(1+\theta) h(k)-0.5 \alpha^{2} p_{k}(1+\theta)^{2} \sigma_{1}^{2}(k)-\sum_{j=1}^{N} q_{k j} p_{j}-\eta p_{k}\right] V_{1}^{2}\right. \\
& +\left[-\alpha p_{k}(1+\theta) b(k)+0.5 \alpha p_{k}(1+\theta)(2+\theta) \sigma_{1}^{2}(k)+2 \sum_{j=1}^{N} q_{k j} p_{j}+2 \eta p_{k}\right] v_{1}+\eta p_{k} \\
& +\sum_{j=1}^{N} q_{k j} p_{j}+\alpha p_{k}(1+\theta) a(k) \prod_{0<t_{k}<t}\left(1+b_{k}\right)^{\lambda} v_{1} y^{\lambda-1-\theta} \\
& +\alpha p_{k}(1+\theta) a(k) \prod_{0<t_{k}<t}\left(1+b_{k}\right)^{\lambda} y^{\lambda-1-\theta} \\
& +0.5 \alpha p_{k}(1+\theta)(2+\theta) \sigma_{2}^{2}(k) \prod_{0<t_{k}<t}\left(1+b_{k}\right)^{2 \theta} y^{\theta-1} \\
& +0.5 \alpha p_{k}(1+\theta)(2+\theta) \sigma_{2}^{2}(k) \prod_{0<t_{k}<t}\left(1+b_{k}\right)^{2 \theta} y^{-2} \\
& \left.+0.5 p_{k} \alpha(\alpha-1)(1+\theta)^{2} \sigma_{2}^{2}(k) \prod_{0<t_{k}<t}\left(1+b_{k}\right)^{2 \theta} y^{-2}\right\} \\
& \leqslant\left(1+v_{1}\right)^{\alpha-2}\left\{-\left[\alpha p_{k}(1+\theta) h(k)-0.5 \alpha^{2} p_{k}(1+\theta)^{2} \sigma_{1}^{2}(k)-\sum_{j=1}^{N} q_{k j} p_{j}-\eta p_{k}\right] v_{1}^{2}\right. \\
& +\left[\alpha p_{k}(1+\theta) \check{b}+0.5 \alpha p_{k}(2+\theta)^{2} \check{\sigma}_{1}^{2}+2 \sum_{j=1}^{N} q_{k j} p_{j}+2 \eta p_{k}\right] v_{1}+\eta p_{k}+\sum_{j=1}^{N} q_{k j} p_{j} \\
& +\alpha p_{k}(1+\theta) \check{a} M^{\lambda} V_{1} y^{\lambda-1-\theta}+\alpha p_{k}(1+\theta) \check{a} M^{\lambda} y^{\lambda-1-\theta}+0.5 \alpha p_{k}(2+\theta)^{2} \check{\sigma}_{2}^{2} M^{2 \theta} y^{\theta-1} \\
& \left.+0.5 \alpha p_{k}(2+\theta)^{2} \check{\sigma}_{2}^{2} M^{2 \theta} y^{-2}+0.5 p_{k} \alpha^{2}(2+\theta)^{2} \check{\sigma}_{2}^{2} M^{2 \theta} y^{-2}\right\} \\
& :=\mathrm{J}(\mathrm{y}, \mathrm{k})
\end{aligned}
$$

We claim that $J(y, k)$ is upper bounded. Without loss of generality, suppose that $\check{\sigma}_{2}^{2}>0$. Set

$$
L_{k}=\min \left\{1,\left(\frac{\alpha p_{k}(1+\theta) h(k)-0.5 \alpha^{2} p_{k}(1+\theta)^{2} \sigma_{1}^{2}(k)-\sum_{j=1}^{N} q_{k j} p_{j}-\eta p_{k}}{\alpha p_{k}(2+\theta)^{2}(1+\alpha) \check{\sigma}_{2}^{2} M^{2 \theta}}\right)^{1 /(2 \theta)}\right\} .
$$

(a) If $y \geqslant L_{k}$, by the definition of $V_{1}$ and the condition $0<\theta \leqslant 1,0<\lambda \leqslant 1+\theta, J(y, k)$ is upper bounded, namely, there exists a positive number $J_{1 k}$ such that $\sup _{y \geqslant L_{k}} J(y, k)<J_{1 k}$.

(b) If $y<\mathrm{L}_{\mathrm{k}}$. For $0<\lambda \leqslant 1+\theta$, we can easily see that there exists a constant $\tau \in(0,1]$ such that $\lambda=\tau(1+\theta)$. Recalling $y<1$, one can see that

$$
y^{\lambda-1-\theta}=y^{\tau(1+\theta)} y^{-(1+\theta)}=V_{1}^{1-\tau}, \quad y^{\theta-1}=y^{2 \theta} y^{-(1+\theta)} \leqslant V_{1}
$$

Note that

$$
y<\left(\frac{\alpha p_{k}(1+\theta) h(k)-0.5 \alpha^{2} p_{k}(1+\theta)^{2} \sigma_{1}^{2}(k)-\sum_{j=1}^{N} q_{k j} p_{j}-\eta p_{k}}{\alpha p_{k}(2+\theta)^{2}(1+\alpha) \check{\sigma}_{2}^{2} M^{2 \theta}}\right)^{1 /(2 \theta)}
$$

then

$$
-0.5\left[\alpha p_{k}(1+\theta) h(k)-0.5 \alpha^{2} p_{k}(1+\theta)^{2} \sigma_{1}^{2}(k)-\sum_{j=1}^{N} q_{k j} p_{j}-\eta p_{k}\right] V_{1}^{2}+0.5 \alpha p_{k}(2+\theta)^{2}(1+\alpha) \check{\sigma}_{2}^{2} M^{2 \theta} y^{-2}<0
$$


Substituting (4.2) and (4.3) into (4.1), we receive

$$
\begin{aligned}
J(y, k) \leqslant & \left(1+v_{1}\right)^{\alpha-2}\left\{-0.5\left[\alpha p_{k}(1+\theta) h(k)-0.5 \alpha^{2} p_{k}(1+\theta)^{2} \sigma_{1}^{2}(k)-\sum_{j=1}^{N} q_{k j} p_{j}-\eta p_{k}\right] V_{1}^{2}\right. \\
& +\left[\alpha p_{k}(1+\theta) \check{b}+0.5 \alpha p_{k}(2+\theta)^{2} \check{\sigma}_{1}^{2}+2 \sum_{j=1}^{N} q_{k j} p_{j}+2 \eta p_{k}\right] V_{1}+\eta p_{k}+\sum_{j=1}^{N} q_{k j} p_{j} \\
& \left.+\alpha p_{k}(1+\theta) \check{a} M^{\lambda} V_{1}^{2-\tau}+\alpha p_{k}(1+\theta) \check{a} M^{\lambda} V_{1}^{1-\tau}+0.5 \alpha p_{k}(2+\theta)^{2} \check{\sigma_{2}^{2}} M^{2 \theta} V_{1}\right\} .
\end{aligned}
$$

By now, we can conclude that if $y<L_{k}$, then there exists a positive number $J_{2 k}$ such that $\sup _{y<L_{k}} J(y, k)<$ $J_{2 k}$. Choose $J=\max _{k \in S}\left\{J_{1 k}, J_{2 k}\right\}$, then $\sup _{y \in \mathbb{R}_{+}, k \in S} J(y, k)<J<\infty$ is obtained. Hence,

$$
p_{k} e^{\eta t} \mathbb{E}\left[\left(1+V_{1}(y(t))\right)^{\alpha}\right] \leqslant p_{k}\left(1+V_{1}(y(0))\right)^{\alpha}+J\left(e^{\eta t}-1\right) / \eta \text {. }
$$

Further,

$$
\limsup _{t \rightarrow+\infty} \mathbb{E}\left[y^{-\alpha(1+\theta)}(t)\right]=\limsup _{t \rightarrow \infty} \mathbb{E}\left[V_{1}^{\alpha}(y(t))\right] \leqslant \limsup _{t \rightarrow \infty} \mathbb{E}\left[\left(1+V_{1}\right)^{\alpha}(y(t))\right] \leqslant J /(\eta \hat{p}) .
$$

Then,

$$
\begin{aligned}
\limsup _{\mathrm{t} \rightarrow+\infty} \mathbb{E}\left[x(t)^{-\alpha(1+\theta)}\right] & =\limsup _{\mathrm{t} \rightarrow+\infty} \mathbb{E}\left[\prod_{0<\mathrm{t}_{k}<t}\left(1+\mathrm{b}_{\mathrm{k}}\right)^{-\alpha(1+\theta)} \mathrm{y}^{-\alpha(1+\theta)}(\mathrm{t})\right] \\
& \leqslant \mathrm{m}^{-\alpha(1+\theta)} \limsup _{\mathrm{t} \rightarrow+\infty} \mathbb{E}\left[\mathrm{y}^{-\alpha(1+\theta)}(\mathrm{t})\right] \\
& \leqslant \mathrm{m}^{-\alpha(1+\theta)} \mathrm{J} /(\eta \hat{\mathrm{p}}):=\widetilde{J} .
\end{aligned}
$$

For any given $\varepsilon>0$, let $\mathrm{H}_{2}=(\varepsilon / \widetilde{J})^{1 /(\alpha(1+\theta))}$, using Chebyshev inequality deduces that

$$
\mathbb{P}\left\{x(t) \leqslant H_{2}\right\}=\mathbb{P}\left\{x^{-\alpha(1+\theta)}(t) \geqslant H_{2}^{-\alpha(1+\theta)}\right\} \leqslant \mathbb{E}\left[x^{-\alpha(1+\theta)}(t)\right] / H_{2}^{-\alpha(1+\theta)}=H_{2}^{\alpha(1+\theta)} \mathbb{E}\left[x^{-\alpha(1+\theta)}(t)\right] .
$$

So, $\lim \sup _{t \rightarrow+\infty} \mathbb{P}\left\{x(t) \leqslant H_{2}\right\} \leqslant \varepsilon$. Then, $\liminf _{t \rightarrow+\infty} \mathbb{P}\left\{x(t) \geqslant H_{2}\right\} \geqslant 1-\varepsilon$ is obtained. As applications of Chebyshev's inequality and Lemma 4.2 , we have $\liminf _{t \rightarrow+\infty} \mathbb{P}\left[x(t) \leqslant H_{1}\right] \geqslant 1-\varepsilon$. Therefore, the stochastic permanence is obtained. This completes the proof.

Remark 4.6. From the conditions and results of Theorem 4.5, we can see that the stochastic permanence of the population has close relations with the impulse and stationary distribution of the Markov chain. In order to make the population survive forever, the impulse cannot be too small or too large, in other words, the impulse must be in a certain range, this is also consistent with the reality.

Remark 4.7. From the conditions of Theorem 4.5, we can see that the property of stochastic permanence of the population has relation with the power of the system. This implies that our model is more general and meaningful.

\section{Numerical simulations}

In this section, we use the Milsteins Method (see [10]) to illustrate our results and the impulsive effects on the population. First we give an example to illustrate the effects of the negative impulse on the populations.

Example 5.1. For model (1.5), the parameters are chosen as follows: $b(r(t))=0.6, a(r(t))=0.4, \sigma_{1}^{2}(r(t))=$ $0.8, \sigma_{2}^{2}(r(t))=1.5, \lambda=0.9, \theta=0.1$. 
By simple computation, we see $h(r(t))=0.6-0.4>0$, by the results of [22], the species will be weakly persistent, in Figure 1 the blue line shows this.

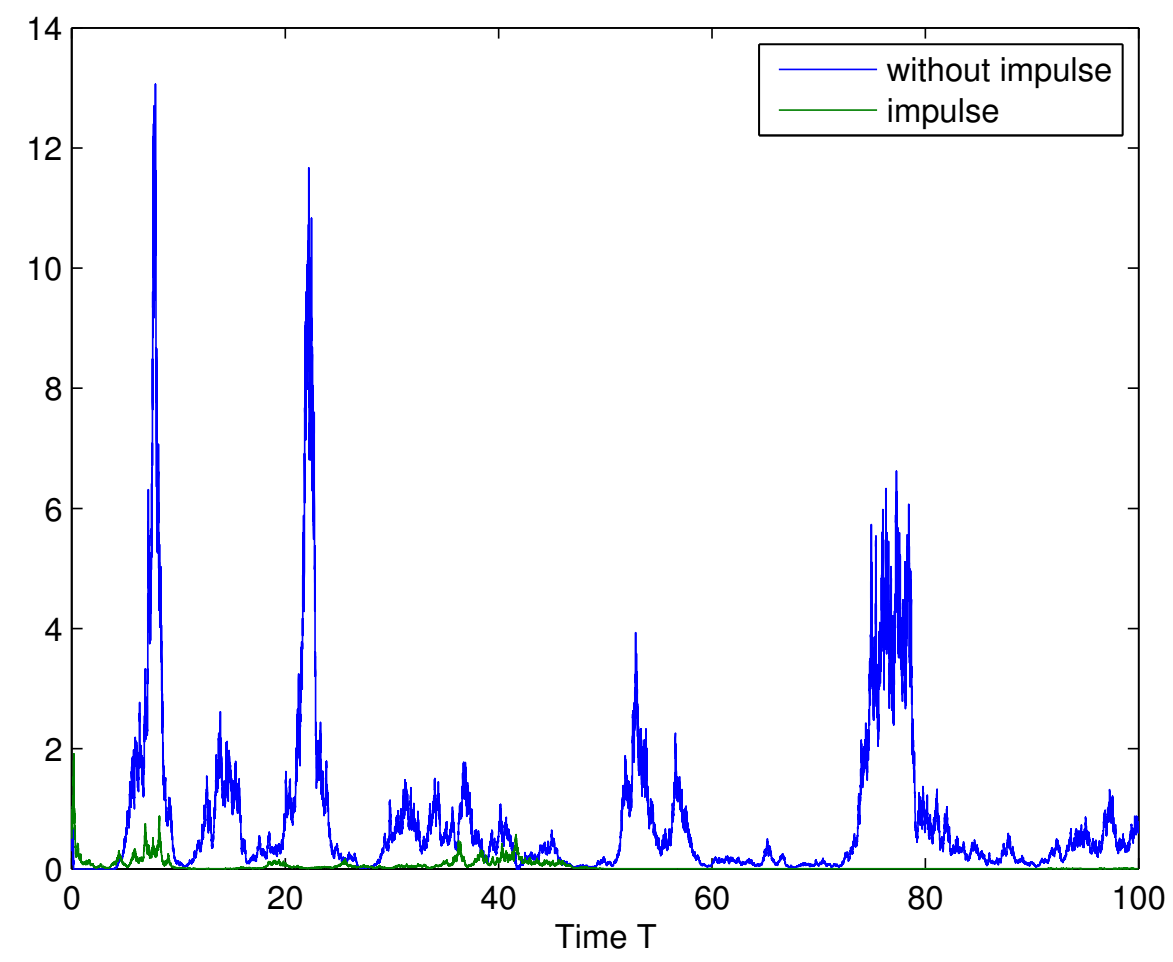

Figure 1: The negative impulse is disadvantageous for the persistence of the species.

Now we take the impulse into account, in Example 5.1, let $b_{k}=e^{-0.6}-1, t_{k}=k$ be negative impulses, then the result changes greatly. By computation, we have $\limsup _{t \rightarrow \infty} t^{-1} \sum_{0<t_{k}<t} \ln \left(1+b_{k}\right)+h(r(t))=$ $-0.4<0$, then species $x(t)$ will be extinct. In Figure 1, the green line illustrates this. This example reveals that the negative impulses are disadvantageous for the ecosystem.

In the following, we present examples to show the regulation of the Markov chain. Here, we consider the Markov chain with two state space $S=\{1,2\}$.

Example 5.2. For model (1.5), choose the initial value $x(0)=0.2$ with the following choice of parameters: $\mathrm{b}(1)=0.3, \mathrm{a}(1)=0.5, \sigma_{1}^{2}(1)=0.8, \sigma_{2}^{2}(1)=5 ; \mathrm{b}(2)=0.5, \mathrm{a}(2)=0.5, \sigma_{1}^{2}(2)=0.6, \sigma_{2}^{2}(2)=3, \mathrm{~b}_{\mathrm{k}}=e^{-\frac{1}{k^{2}}}-$ $1, t_{k}=k, \lambda=0.9, \theta=0.1$.

By computation, we have $h(1)=b(1)-0.5 \sigma_{1}^{2}(1)=0.3-0.4<0, h(2)=b(2)-0.5 \sigma_{1}^{2}(2)=0.5-0.3>0$, by Theorems 3.3 and 3.7, we conclude that:

in state 1, the population will be extinct; and in state 2 , the population will be weakly persistent. The state 1 can be regarded as the "bad" state, and the state 2 can be considered as the "good" state. First, we take the generator of the Markov chain as $\mathrm{Q}=\left(\begin{array}{cc}-1 & 1 \\ 4 & -4\end{array}\right)$, by (1.3) the unique stationary distribution $\pi$ of $r(t)$ is expressed by $\pi=\left(\pi_{1}, \pi_{2}\right)=(4 / 5,1 / 5)$. Then $\bar{h}=\limsup _{t \rightarrow \infty} t^{-1} \sum_{0<t_{k}<t} \ln \left(1+b_{k}\right)+$ $\sum_{i=1}^{2} \pi_{i} h(i)=-0.04<0$, by Theorem 3.3, the species will be extinct, see Figure 2 .

Now choose the generator of the Markov chain to be $Q=\left(\begin{array}{cc}-2 & 2 \\ 1 & -1\end{array}\right)$, so the corresponding stationary distribution is denoted by $\pi=\left(\pi_{1}, \pi_{2}\right)=(1 / 3,2 / 3)$. Then we have $\tilde{h}=\sum_{i=1}^{2} \pi_{i} h(i)=0.1>0$, by Theorem 4.5, the population will be stochastically permanent, see Figure 3. 

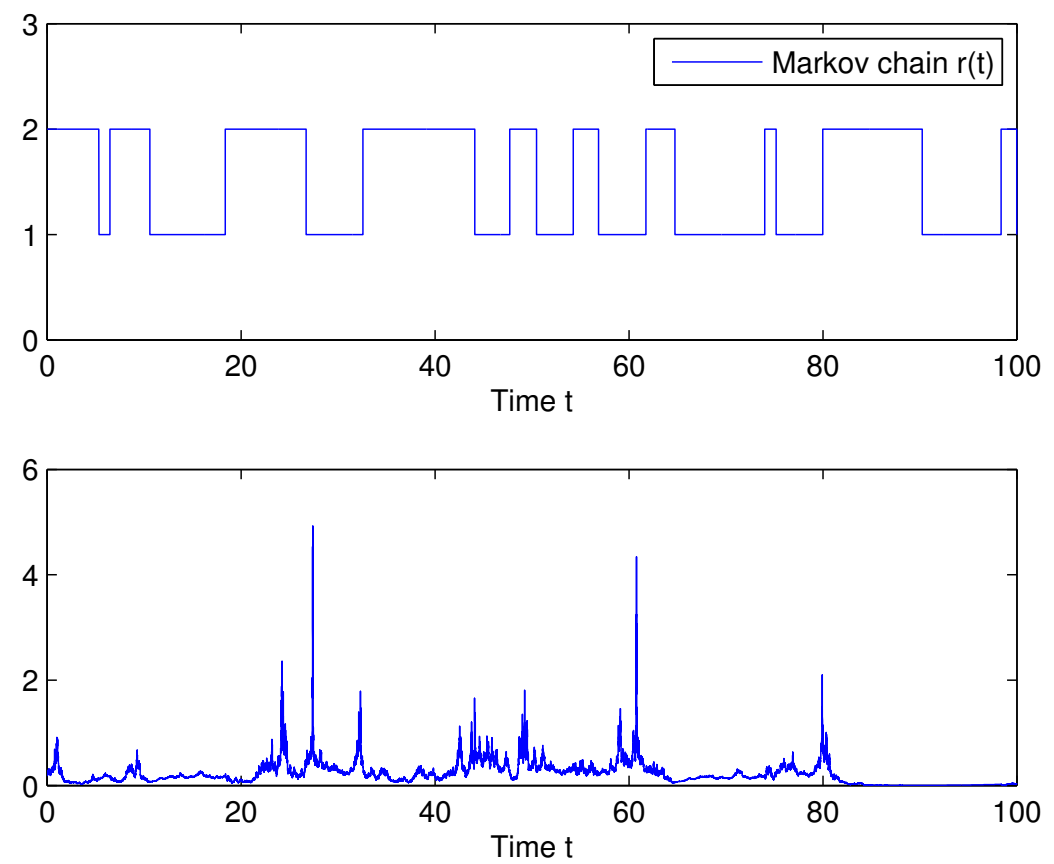

Figure 2: The stationary distribution $\pi=\left(\pi_{1}, \pi_{2}\right)=(4 / 5,1 / 5)$.
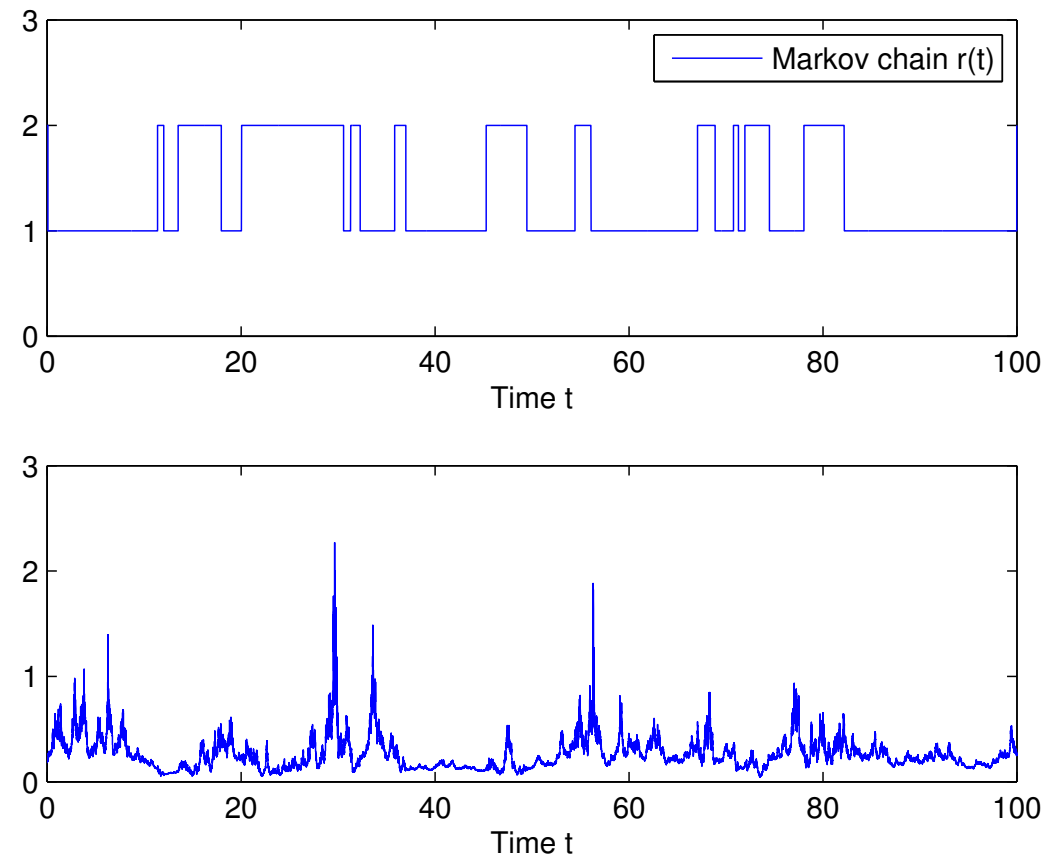

Figure 3: The stationary distribution $\pi=\left(\pi_{1}, \pi_{2}\right)=(1 / 3,2 / 3)$. 


\section{Conclusions and further remarks}

This paper is concerned with the stochastic Gilpin-Ayala model under regime switching with impulsive perturbations. We consider several asymptotic properties and present sufficient conditions for stochastic permanence and extinction. Our key contributions are as follows.

(A) The model is new and more general. By now, as our knowledge is concerned, the extinction and stochastic permanence of the model under regime switching with impulse has little been reported. In this paper, we discuss in detail the effects of the Markov chain and impulse on the dynamics.

(B) The critical value among the extinction, non-persistence in the mean and weak persistence is given.

(C) Our results demonstrate that the long time behaviors of the model have close relations with the impulse and stationary distribution of the Markov chain, see Remarks 3.5, 3.8, 3.9, 3.10, 3.12, 4.6.

(D) From our results we can see that the Markovian switching plays important roles in the model, it can regulate the overall property of the system, see Remarks 3.12, 4.6.

Some interesting topics deserve further consideration. In this paper, we consider the single-species model. One may investigate some n-species models, it may be more difficult, about biological multiple models, readers can refer the references [20, 21].

\section{Acknowledgment}

This research was partially supported by the Fundamental Research Funds for the Central Universities, No.27R1510041A, and Natural Science Foundation of China, No.11526192, No.11401136, and Natural Science Foundation of Shandong Province, No.ZR2014AQ010, and Natural Scientific Research Innovation Foundation in Harbin Institute of Technology (HIT.NSRIF.2015103), and Scientific Research Foundation of Harbin Institute of Technology at Weihai (HIT(WH)201420).

\section{References}

[1] S. Ahmad, I. M. Stamova, Asymptotic stability of competitive systems with delays and impulsive perturbations, J. Math. Anal. Appl., 334 (2007), 686-700. 1

[2] W. J. Anderson, Continuous-time Markov chains, An applications-oriented approach, Springer Series in Statistics: Probability and its Applications, Springer-Verlag, New York, (1991). 1

[3] D. Bănov, P. Simeonov, Impulsive differential equations: periodic solutions and applications, Pitman Monographs and Surveys in Pure and Applied Mathematics, Longman Scientific \& Technical, Harlow; copublished in the United States with John Wiley \& Sons, Inc., New York, (1993). 1

[4] F.-D. Chen, Some new results on the permanence and extinction of nonautonomous Gilpin-Ayala type competition model with delays, Nonlinear Anal., 7 (2006), 1205-1222. 1

[5] N. H. Du, R. Kon, K. Sato, Y. Takeuchi, Dynamical behavior of Lotka-Volterra competition systems: non-autonomous bistable case and the effect of telegraph noise, J. Comput. Appl. Math., 170 (2004), 399-422. 1

[6] M. Fan, K. Wang, Global periodic solutions of a generalized n-species Gilpin-Ayala competition model, Comput. Math. Appl., 40 (2000), 1141-1151. 1

[7] T. C. Gard, Persistence in stochastic food web models, Bull. Math. Biol., 46 (1984), 357-370. 1

[8] T. C. Gard, Stability for multispecies population models in random environments, Nonlinear Anal., 10 (1986), 1411-1419. 1

[9] M.-X. He, F.-D. Chen, Dynamic behaviors of the impulsive periodic multi-species predator-prey system, Comput. Math. Appl., 57 (2009), 248-265. 1

[10] D. J. Higham, An algorithmic introduction to numerical simulation of stochastic differential equations, SIAM Rev., 43 (2001), 525-546. 5

[11] J. Hou, Z.-D. Teng, S.-J. Gao, Permanence and global stability for nonautonomous N-species Lotka-Valterra competitive system with impulses, Nonlinear Anal. Real World Appl., 11 (2010), 1882-1896. 1

[12] D.-Q. Jiang, N.-Z. Shi, A note on nonautonomous logistic equation with random perturbation, J. Math. Anal. Appl., 303, (2005), 164-172. 1

[13] D.-Q. Jiang, N.-Z. Shi, X.-Y. Li, Global stability and stochastic permanence of a non-autonomous logistic equation with random perturbation, J. Math. Anal. Appl., 340 (2008), 588-597. 1, 3.2 
[14] V. Lakshmikantham, D. D. Bă̌nov, P. S. Simeonov, Theory of impulsive differential equations, Series in Modern Applied Mathematics, World Scientific Publishing Co., Inc., Teaneck, NJ, (1989). 1

[15] X.-Y. Li, D.-Q. Jiang, X.-R. Mao, Population dynamical behavior of Lotka-Volterra system under regime switching, J. Comput. Appl. Math., 232 (2009), 427-448.

[16] C.-X. Li, J.-T. Sun, Stability analysis of nonlinear stochastic differential delay systems under impulsive control, Phys. Lett. A, 374 (2010), 1154-1158. 1

[17] C.-X. Li, J.-T. Sun, R.-Y. Sun, Stability analysis of a class of stochastic differential delay equations with nonlinear impulsive effects, J. Franklin Inst., 347 (2010), 1186-1198.

[18] C.-X. Li, J.-P. Shi, J.-T. Sun, Stability of impulsive stochastic differential delay systems and its application to impulsive stochastic neural networks, Nonlinear Anal., 74 (2011), 3099-3111. 1

[19] B.-S. Lian, S.-G. Hu, Asymptotic behaviour of the stochastic Gilpin-Ayala competition models, J. Math. Anal. Appl., 339 (2008), 419-428. 1

[20] M. Liu, C.-Z. Bai, Analysis of a stochastic tri-trophic food-chain model with harvesting, J. Math. Biol., 73 (2016), 597-625. 6

[21] M. Liu, C.-Z. Bai, Dynamics of a stochastic one-prey two-predator model with Léry jumps, Appl. Math. Comput., 284 (2016), 308-321. 6

[22] M. Liu, K. Wang, Asymptotic properties and simulations of a stochastic logistic model under regime switching, Math. Comput. Modelling, 54 (2011), 2139-2154. 1, 2, 3.1, 3.5, 5

[23] M. Liu, K. Wang, Asymptotic properties and simulations of a stochastic logistic model under regime switching II, Math. Comput. Modelling, 55 (2012), 405-418. 1, 4

[24] M. Liu, K. Wang, Dynamics and simulations of a logistic model with impulsive perturbations in a random environment, Math. Comput. Simulation, 92 (2013), 53-75. 1, 1, 3.9

[25] M. Liu, K. Wang, Asymptotic behavior of a stochastic nonautonomous Lotka-Volterra competitive system with impulsive perturbations, Math. Comput. Modelling, 57 (2013), 909-925. 1

[26] M. Liu, K. Wang, On a stochastic logistic equation with impulsive perturbations, Comput. Math. Appl., 63 (2012), 871-886. 1

[27] M. Liu, K. Wang, Dynamics of a two-prey one-predator system in random environments, J. Nonlinear Sci., 23 (2013), 751-775. 3

[28] M. Liu, K. Wang, Persistence and extinction in stochastic non-autonomous logistic systems, J. Math. Anal. Appl., 375 (2011), 443-457. 3.11

[29] Q. Luo, X.-R. Mao, Stochastic population dynamics under regime switching, J. Math. Anal. Appl., 334 (2007), 69-84. 4

[30] X.-R. Mao, G. G. Yin, C.-G. Yuan, Stabilization and destabilization of hybrid systems of stochastic differential equations, Automatica J. IFAC, 43 (2007), 264-273.

[31] X.-R. Mao, C.-G. Yuan, Stochastic differential equations with Markovian switching, Imperial College Press, London, (2006). 4

[32] R. Sakthivel, J. Luo, Asymptotic stability of nonlinear impulsive stochastic differential equations, Statist. Probab. Lett., 79 (2009), 1219-1223. 1

[33] M. Slatkin, The dynamics of a population in a Markovian environment, Ecol., 59 (1978), 249-256. 1

[34] Y. Takeuchi, N. H. Du, N. T. Hieu, K. Sato, Evolution of predator-prey systems described by a Lotka-Volterra equation under random environment, J. Math. Anal. Appl., 323 (2006), 938-957. 1

[35] R.-H. Wu, X.-L. Zou, K. Wang, Asymptotic properties of a stochastic Lotka-Volterra cooperative system with impulsive perturbations, Nonlinear Dynam., 77 (2014), 807-817. 1

[36] J.-R. Yan, Stability for impulsive delay differential equations, Nonlinear Anal., 63 (2005), 66-80. 1 